\title{
A Study on Virtual Market for Pareto Optimal Mediation in Economic Society
}

\author{
Toshiya Kaihara \\ Graduate School of Science and Technology \\ Kobe University \\ Nada, Kobe, 657-8501, Japan \\ kaihara@cs.kobe-u.ac.jp
}

\author{
Susumu Fujii \\ Computer and Systems Engineering \\ Kobe University \\ Nada, Kobe, 657-8501, Japan \\ fujii@cs.kobe-u.ac.jp
}

\begin{abstract}
Market-oriented programming is a new approach to design and implementation of resource allocation mechanisms in computer systems. It has its roots in different disciplines, such as economics and computer science. In this paper we construct a Walrasian type virtual market, and try to confirm Pareto optimality in our market model by comparing the solutions with conventional analytic approaches. The main conclusion of the paper is that there are some potential pitfalls of Walrasian virtual market, but when used with care it provides a highly natural and efficient means for generic resource allocation problems with using computational market.
\end{abstract}

\section{Introduction}

Market-oriented programming is a new approach to design and implementation of resource allocation mechanisms in computer systems [1]. It has its roots in different disciplines, such as economics and computer science, in particular the area of multi-agent systems. Market-oriented programming is seen as a programming paradigm based on abstractions such as prices and demands. Concepts, terminology and theory from microeconomics form the foundations of the paradigm. Central aspects of these foundations are investigated and some new insights are presented. Furthermore, some relations between conventional optimization/resource allocation approaches and market-oriented programming are described in this paper.

There are our previous researches to apply market-oriented programming into resource allocation problems $[2,3]$. And the Pareto optimality in market-oriented programming was left into microeconomics, and never tried to be proved in these approaches using multi-agent programming. In this paper we construct a Walrasian type virtual market, that is a principal market model in microeconomics, and try to confirm the Pareto optimality in our market model by comparing the solutions with conventional analytic approaches, such as $\varepsilon$ constraint method and fixed-point algorithm.

The main conclusion of the paper is that there are some potential pitfalls of Walrasian virtual market, but when used with care it provides a highly natural and efficient means for generic resource allocation problems with using computational market.

\section{Walrasian virtual market}

There exists a market-oriented programming to construct a computational market (i. e. virtual market), which consists of several heterogeneous agents [1]. Agent activities in terms of products required and supplied are defined so as to reduce an agent' $\mathrm{s}$ decision problem to evaluate the tradeoffs of acquiring different products in the market-oriented programming. These tradeoffs are represented in terms of market prices, which define common scale of value across the various products. The problem for designers of computational markets is to specify the mechanism by which agent interactions determine prices [2].

Market-oriented programming is the general approach of deriving solutions to distributed 
resource allocation problems by computing the competitive equilibrium of an artificial economy. It involves an iterative adjustment of prices based on the reactions of the agent in the market. General concept of the negotiation mechanism in market-oriented programming is shown in figure 1. Definitions of the virtual market are based on general equilibrium concept in perfect competitive market, and that means it satisfies a necessary condition of Walrasian type virtual market.

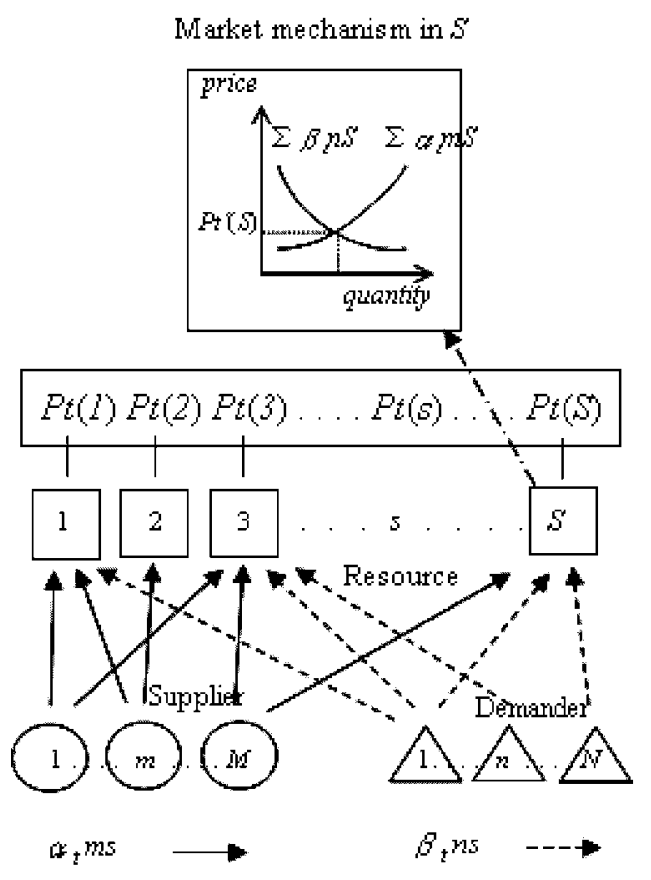

Figure 1: Market-oriented programming

Supply / demand functions represent agent's willingness to sell / buy resources, respectively. They are defined as the relationship between price and quantity of the trading resource. Let $P t(s)$ be the price of resource s at time $t . \alpha_{t} m s$ and $\beta_{t} n s$ represent the supply function of supplier $m$ on resource $s$ at time $t$ and the demand function of demander $n$ on resource $s$ at time $t$, respectively. The bidding mechanism computes an equilibrium price in each separate market. It involves an iterative adjustment of prices based on reactions of agents in the market. Agent $s$ submits supply and demand functions $\left(\alpha_{t} m s\right.$ and $\left.\beta_{t} n s\right)$ and the auc- tion adjusts individual prices to clear, rather than adjusting the entire price vector by some increment. The mechanism associates an auction with each distinct resource. Agents act in the market by submitting bids to auctions. In this paper bids specify a correspondence between prices and quantities of the resource that the agent offers to demand or supply as a basic study. Given bids from all interested agents, the auction derives a market-clearing price.

\section{Economic agent}

We describe demand agent (i.e. consumer) as $c_{m}(m=1,2, \cdots, M)$, and supply agent (i.e. producer $)$ as $s_{n}(n=1,2, \cdots, N)$. The number of kind of goods is assumed as $I$ in our Walrasian VM.

\subsection{Demand agent \\ 3.1.1 Demand utility}

Suppose demand agent $c_{m}$ has utility function $u^{c_{m}}$, which is described with in equation (1). In this equation $x_{i}^{c_{m}}$ represents the demand quantity for resource $i$ :

$$
\begin{gathered}
u^{c_{m}}=a^{c_{m}} \prod_{i=1}^{I}\left(x_{i}^{c_{m}}\right)^{b_{i}^{c_{i}}} \\
\text { where } \sum_{i=1}^{I} b_{i}^{c_{m}}=1 \quad\left(0<a^{c_{m}}, b_{i}^{c_{m}}\right)
\end{gathered}
$$

In this paper we adopt Cobb-Douglas function [4] as a demand function described in equation (1), because the Cobb-Douglas function is one of the primitive functions in microeconomics, which handles economical scale in the market by index constant $b$.

\subsubsection{Budget}

Budget of demand agent $c_{m}$ is formulated by initial quantity of resource $(i): e_{i}^{c_{m}}$, and their price: $p_{i}$ as follows:

$$
B^{c_{m}}=\sum_{i=1}^{I} p_{i} e_{i}^{c_{m}}+r^{c_{m}}
$$

In this equation $r^{c_{m}}$ represents supplier's profit, which suppliers return to demanders under zero-profit conditions in the general equilibrium theory [5]. 


\subsubsection{Bidding functions}

Demand agents send their bid to their target resources in the market, and the bid is formulated as demand function. The function is obtained as the optimal solution as maximising problem of equation (1) under the constraints described in equation (2). The following demand function is calculated by Lagrange's method of (indeterminate) multiplier in this research.

$$
x_{i}^{c_{m}}\left(p_{i}\right)=\frac{b_{i}^{c_{m}} B^{c_{m}}}{p_{i}} \quad i=1,2, \ldots, I
$$

We assume demander $c_{m}$ supplies all the initial resources into the market according to the principle of microeconomics, and demander's supply function of resource $j$ is defined as follows:

$$
y_{j}^{c_{m}}=e_{j}^{c_{m}}
$$

\subsection{Supply agent}

\subsubsection{Production function}

As described in demand agent definitions, Cobb-Douglas function is basic functions which handles economical scale in the market easily. In microeconomics production function is assumed to be concave function, and that means market prices are established at a predictable level in the general equilibrium theory in concave shape production function.

We also formulate production function of supply agent sn to resource $j$ as Cobb-Douglas function to satisfy the assumption, shown as equation (5). Cobb-Douglas function is defined as a concave function in $0<\beta<1$ in this equation.

$$
y_{j}^{s_{n}}=\alpha^{s_{n}}\left(x_{i}^{s_{n}}\right)^{\beta^{s_{n}}} \quad\left(0<\alpha^{s_{n}}, 0<\beta^{s_{n}}<1\right)
$$

\subsubsection{Profit}

According to microeconomics assumption, supply agents have no initial resources. They can earn their profit $\pi^{s_{n}}$ by producing value added resources from purchased resources. The profit function is defined as follows:

$$
\pi^{s_{n}}=p_{j} y_{j}^{s_{n}}-p_{i} x_{i}^{s_{n}}
$$

\subsubsection{Bidding functions}

Supply agents send supply functions to production resources, and demand functions to purchase resources, respectively. They maximise their profit by solving maximising problem of equation (6) under the constraint in equation (5). We also solve the problem by Lagrange' s method of multiplier in this research, and obtain the following demand function and supply function in equation (7) and (8), respectively:

$$
\begin{gathered}
x_{i}^{s_{n}}\left(p_{i}\right)=\left(\frac{p_{i}}{\alpha^{s_{n}} \beta^{s_{n}} p_{j}}\right)^{\frac{1}{\beta^{s_{n}-1}}} \\
y_{j}^{s_{n}}\left(p_{j}\right)=\left(\frac{\left(p_{i}\right)^{\beta^{s_{n}}}}{\alpha^{s_{n}}\left(\beta^{s_{n}}\right)^{\beta^{s_{n}}}\left(p_{j}\right)^{\beta^{s_{n}}}}\right)^{\frac{1}{\beta^{s_{n}-1}}}
\end{gathered}
$$

\section{Analytic approach}

We demonstrate the proposed algorithm successfully calculates Pareto optimal solutions by comparing VM solutions with an analytic approaches, named fixed-point algorithm. We will explain this analytic approach briefly below.

Scarf showed that how to compute an approximate Walras equilibrium and proposed a general algorithm for the calculation of a fixed point of a correspondence [6]. This algorithm, named Scarf's algorithm, has been surprisingly efficient to find a general equilibrium that was guaranteed to converge, though does not permit a gradual improvement in the degree of approximation of the solution.

The Scarf's algorithm applies a procedure to the problem of computing a fixed point of mapping of the unit simples into itself, a mapping whose existence is established by fixed point theorem. In using Scarf's algorithm to find such a fixed point, the unit simplex is divided into a finite number of smaller simplices, each defined by $I$ vertices that are each associated with a label (figure 2). Each vertex is labelled with the index number of goods, which has the maximum value in market excess demand. The algorithm tries to seek the subdivided simplex whose vertices have all labels without any duplicates, and Pareto optimal solution must be 
inside the subdivided simplex. The market excess demand of goods $i$ at the vertex is defined as the next equation:

$$
E_{i}=\left(\sum_{m=1}^{M} x_{i}^{c_{m}}+\sum_{n=1}^{N} x_{i}^{s_{n}}\right)-\left(\sum_{m=1}^{M} y_{i}^{c_{m}}+\sum_{n=1}^{N} y_{i}^{s_{n}}\right)
$$

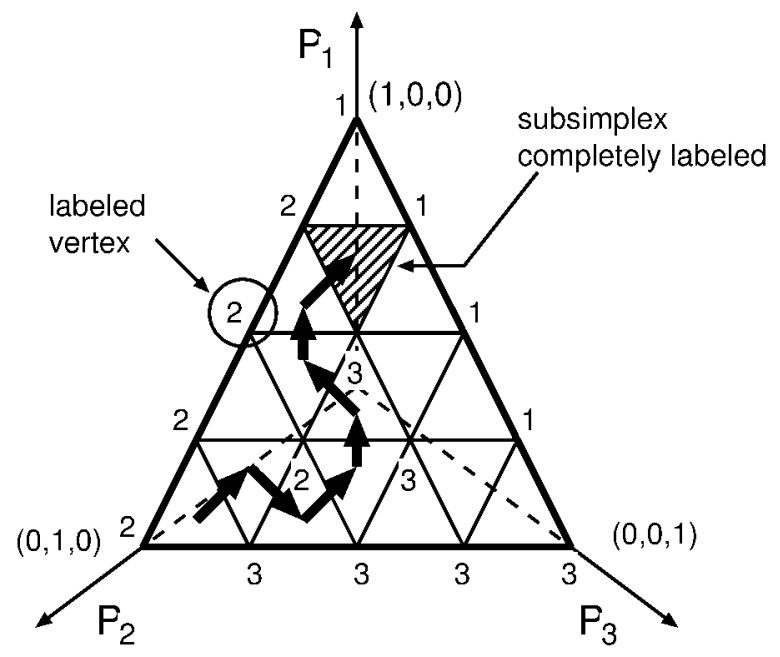

Figure 2: An example of 2-dimentional unit simplex

\section{Experimental results}

Experimental 2-producer-2-consumer market is illustrated in figure 3 . In this market 2 kinds of goods, goods 1 and goods 2 , are also traded.

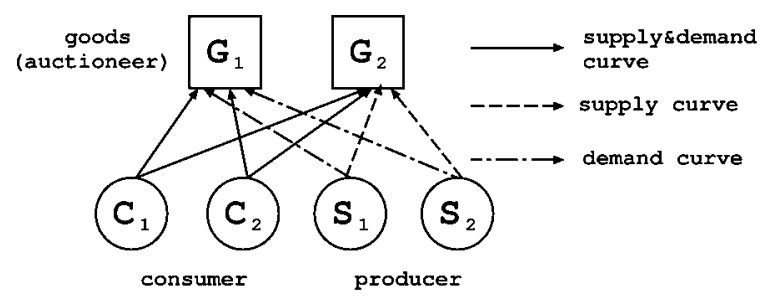

Figure 3: Virtual market in trade market

Experimental values of each parameter (i.e. initial value set) in the model are shown in Table 1(consumer agent) and Table 2 (producer agent). Consumer agents send their bids as supply \& demand functions to both the goods homogeneously. Producer agents send their demand \& supply function to goods $1 \&$ goods 2 , respectively. That means they both produce goods 2 from goods 1 in this market.

Table 1: Initial conditions of consumers

\begin{tabular}{|c|c|c|c|}
\hline Agent & $\begin{array}{c}\text { Utility } \\
\text { function }\left(u^{c_{m}}\right)\end{array}$ & $\begin{array}{c}\text { Endowment } \\
\left(e_{1}^{c_{m}}, e_{2}^{c_{m}}\right)\end{array}$ & $\begin{array}{c}\text { Utility } \\
\text { value }\end{array}$ \\
\hline$c_{1}$ & $1.0\left(x_{1}^{c_{1}}\right)^{0.8}\left(x_{2}^{c_{1}}\right)^{0.2}$ & $(30.0,80.0)$ & 36.50 \\
$c_{2}$ & $1.0\left(x_{1}^{c_{2}}\right)^{0.3}\left(x_{2}^{c_{2}}\right)^{0.7}$ & $(70.0,20.0)$ & 29.12 \\
\hline
\end{tabular}

Table 2: Initial conditions of producers

\begin{tabular}{|c||c|c|c|}
\hline Agent & $\begin{array}{c}\text { Input } \\
\text { goods }\end{array}$ & $\begin{array}{c}\text { Output } \\
\text { goods }\end{array}$ & $\begin{array}{c}\text { Production } \\
\text { function }\end{array}$ \\
\hline$s_{1}$ & goods 1 & goods 2 & $y_{2}^{s_{1}}=2.0\left(x_{1}^{s_{1}}\right)^{0.7}$ \\
$s_{2}$ & goods 1 & goods 2 & $y_{2}^{s_{2}}=3.0\left(x_{1}^{s_{2}}\right)^{0.5}$ \\
\hline
\end{tabular}

Simulation results on price and trade changes of each goods in the Walrasian VM are shown in figure 4 , and 5 , respectively.

In this model we obtained equilibrium price vector $\hat{p}=\left(\hat{p_{1}}, \hat{p_{2}}\right)$ as follows:

Equilibrium price vector:

$$
\hat{p}=(0.95673,0.98543)
$$

Normalised equilibrium price vector:

$$
\hat{p}=(0.49261,0.50789)
$$

And final resource allocation in consumer agent and producer agent are shown in table 3 and 4 , respectively.

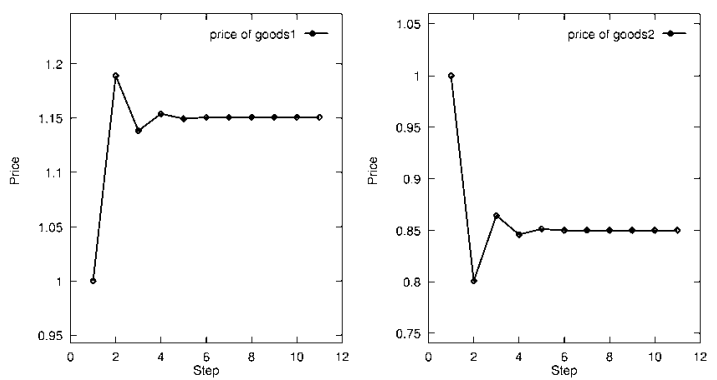

Figure 4: Price transition in trade market

It has been observed that the total consumer's utility is increased in table 3 compared 

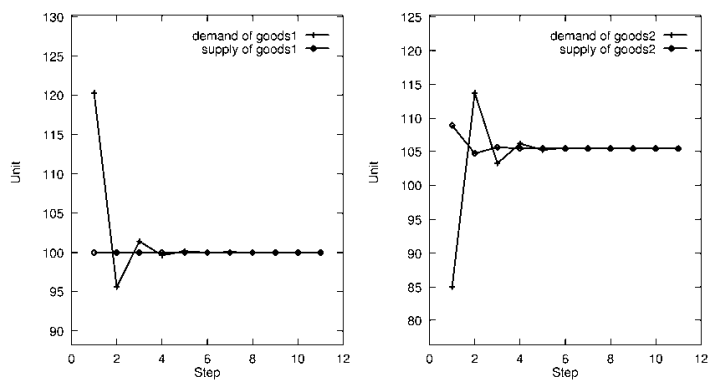

Figure 5: Supply \& demand transition in trade market

with table 2 , because the producer's profit is returned to consumer agents in this market under the zero-profit conditions in the general equilibrium theory. The utility of agent $c_{1}$ is increased by $17.2 \%$ especially. That is because of the agent's preference to goods 2 as well as the zero-profit conditions. In this market all the producer agents supply goods 2 , and that increases the utility of agent $c_{1}$. On the other hand, the utility of agent $c_{2}$ is slightly decreased, because it becomes slightly difficult to get both goods due to the stronger supply flow from producer agents to agent $c_{1}$.

Table 3: Equilibrium conditions of consumers in trade market

\begin{tabular}{|c||c|c|}
\hline Agent & $\begin{array}{c}\text { Consumption } \\
\left(x_{1}^{c_{m}}, x_{2}^{c_{m}}\right)\end{array}$ & $\begin{array}{c}\text { Utility } \\
\text { value }\end{array}$ \\
\hline$c_{1}$ & $(71.96,24.35)$ & 57.94 \\
$c_{2}$ & $(25.69,81.14)$ & 57.46 \\
\hline
\end{tabular}

Table 4: Equilibrium conditions of producers

\begin{tabular}{|c|c|c|c|}
\hline Agent & Production & Input & Profit \\
\hline$s_{1}$ & 2.16 & 1.12 & 0.55 \\
$s_{2}$ & 3.32 & 1.23 & 1.41 \\
\hline Total & 5.48 & 2.35 & 1.96 \\
\hline
\end{tabular}

We applied fixed point algorithm in this market model to confirm Pareto optimality of the VM solutions. We calculated a fixed point of mapping of the unit simples into itself, a mapping whose existence is established by fixed point theorem. In using Scarf' s algorithm to find such a fixed point, the unit simplex is divided into a finite number of smaller simplices (i.e. grid size). In this paper the grid size is set to 100,000 for the precise comparison.

We obtained equilibrium price vector $\hat{p}=$ $\left(\hat{p_{1}}, \hat{p_{2}}\right)$ by the fixed point algorithm as follows:

\section{Equilibrium price vector:}

$$
\hat{p}=(0.49262,0.50789)
$$

It is obvious that the equilibrium price set obtained by Walrasian VM is almost equivalent to the one from the fixed point algorithm, and that means VM solutions have been confirmed to be converged into Pareto optimal. The small difference is caused by the grid size of the fixed point algorithm.

We compared the calculation time between VM approach and fixed point algorithm. CPU consumption time (second) for each approach in this market model is as table 5 .

Table 5: CPU consumption time

\begin{tabular}{|c|c|}
\hline Method & CPU time (second) \\
\hline VM & 0.0017 \\
Fixed point algorithm & 12.025 \\
\hline
\end{tabular}

VM approach is obviously more than 7,000 times as fast as the analytic approach in this model. It has also been proved that the proposed VM based approach is much more practical in terms of calculation time to obtain Pareto optimal solutions in resource allocation problems.

By the computer simulation, we have confirmed that Walrasian VM takes advantage of the market analogy into resource allocation problem, and that leads to effective search of Pareto optimal solution for supply chain management.

\section{Conclusions}

Market-oriented programming is a new approach to design and implementation of resource allocation mechanisms in computer systems, and we newly proposed a Walrasian Virtual Market (VM) approach with microeconomics based market-oriented programming. 
Firstly we mentioned our general concept to apply VM into resource allocation problems, and explained general idea of Walrasian market model in economics. Then central aspects of market-oriented programming are investigated and some new insights are presented. After a brief explanation of the analytical approaches, named fixed-point algorithm, we defined agent behaviour based on Walrasian market-oriented programming. As a basic study, we analysed its Pareto optimality by computer simulation experiments, and it has been confirmed that our approach is efficient both in Pareto optimality and calculation performance.

\section{References}

[1] Wellman M. P., A Market-Oriented Programming Environment and its Application to Distributed Multi-commodity Flow Problems, ICMAS-96, pp385-392, 1996.

[2] Kihara, T., Supply chain management based on market Mechanism in virtual enterprise, Infrastructures for Virtual Enterprises, Kluwer Academic Publishers, Boston, pp.399-408, 1999.

[3] Kihara, T., Supply Chain Management with Market Economics, International Journal of Production Economics, Elsevier Science, Vol. 73, Issue 1, pp.5-14, 2001.

[4] Kreps, D. M., A Course in Microeconomic Theory, Harvester Wheatsheaf, New York, 1990.

[5] Shoven, J. B. and J. Whalley, Applying General Equilibrium, Cambridge University Press, New York, 1992.

[6] Scarf, H. E., The computing of Economics Equilibria, New Heaven, Yale University Press, 1973. 